\title{
Calvarial bone defects in ovariectomised rats treated with mesenchymal stem cells and demineralised freeze-dried bone allografts
}

\author{
E.T. Kadiroğlu' ${ }^{1}$ M.E. Akbalık², E. Karaöz ${ }^{3}$, B.E. Kanay4 ${ }^{4}$ A. Dağ ${ }^{5}$, M.A. Ketani ${ }^{6}$, \\ E.G. Eroğlu' ${ }^{7}$ E. Uysal ${ }^{8}$, M.C. Tuncer ${ }^{9}$ \\ 'Department of Periodontology, Faculty of Dentistry, Dicle University, Diyarbakır, Turkey \\ ${ }^{2}$ Department of Histology and Embryology, Faculty of Veterinary Medicine, Dicle University, Diyarbakır, Turkey \\ ${ }^{3}$ Department of Histology and Embryology, Faculty of Medicine, University of Istinye, Istanbul, Turkey \\ ${ }^{4}$ Department of Surgery, Faculty of Veterinary Medicine, Dicle University, Diyarbakır, Turkey \\ ${ }^{5}$ Department of Periodontology, Faculty of Dentistry, Dicle University, Diyarbakır, Turkey \\ ${ }^{6}$ Department of Histology and Embryology, Faculty of Veterinary Medicine, Dicle University, Diyarbakır, Turkey \\ 'Oral and Dental Health Centre, Van, Turkey \\ ${ }^{8}$ Diyarbakır Vocational School of Technical Science, Dicle University, Diyarbakır, Turkey \\ ${ }^{9}$ Department of Anatomy, Faculty of Medicine, Dicle University, Diyarbakır, Turkey
}

[Received: 6 November 2019; Accepted: 14 December 2019]

Background: The aim of the study was to investigate the ability of a combination of bone marrow mesenchymal stem cells (BM-MSCS) with and without demineralised freeze-dried bone allografts (DFDBAs) to induce bone regeneration in calvarial defects in ovariectomised rats.

Materials and methods: Critical size defects were filled with a combination of DFDBAs and BM-MSCs or BM-MSCs alone. Eight weeks after calvarial surgery, the rats were sacrificed. The samples were analysed histologically and immunohistochemically.

Results: No difference was observed in vascularisation between groups C1 (animals with cranial defect only, control group) and $\mathrm{O} 1$ (animals with cranial defect only, ovariectomy group). Intramembranous ossification was observed at a limited level in groups $\mathrm{C} 2$ (animals with cranial defect with MSCs, control group) and $\mathrm{O} 2$ (animals with cranial defect with MSCS, ovariectomy group) compared to C1 and 01 . In group C3 (animals with DFDBAs with MSCs, control group), the fibrous structures of the matrix became compact as a result of a bone graft having been placed in the cavity, but in group 03 (animals with DFDBAs with MSCs, ovariectomy group), the fibrous tissue was poorly distributed between the bone grafts for the most parts.

Conclusions: We conclude that the insertion of BM-MSCs enhances bone healing; however, the DFDBA/BM-MSC combination has little effect on overcoming impaired bone formation in ovariectomised rats. (Folia Morphol 2020; 79, 4: 720-735)

Key words: bone healing, bone marrow mesenchymal stem cells (BM-MSCs), demineralised freeze-dried bone allografts (DFDBAs), ovariectomy, calvarial defect

Address for correspondence: M.C. Tuncer, Professor, PhD (The Chief of Anatomy Department), Dicle University, Medical School, Department of Anatomy, 21280, Diyarbakır, Turkey, tel: +90 412 2488001, ext. 4539 (faculty room), fax: +904122488440 , mobile phone: +90 532 2744926, e-mail: drcudi@hotmail.com 


\section{INTRODUCTION}

Oestrogen deficiency is an important cause of postmenopausal bone loss. It leads to an imbalance in osteoblast and osteoclast number. The effect of oestrogen on bone metabolism is mediated by proinflammatory cytokines. In oestrogen deficiency conditions, monocytes and macrophages produce large amounts of the cytokines interleukin (IL)-1, IL-6, tumour necrosis factor- $\alpha$, granulocyte-macrophage colony-stimulating factor, macrophage colony-stimulating factor, and prostaglandin- $E_{2}$ that stimulate mature osteoclasts, and consequently induce bone resorption [25].

Several studies have suggested that there is a relationship between systemic low bone density and the onset of periodontitis, which is characterised by the loss of connective tissue and alveolar bone, and risk factors, such as genetics, environmental factors, hormone levels, smoking and diabetes, associated with osteoporosis. Authors have also remarked that osteoporosis may have an effect on the progression of periodontitis via the loss of bone mineral density in the maxilla and mandible in postmenopausal women [12, 24].

Diminished bone density enhances the destruction of alveolar bone, which complicates bone regenerative procedures [19].

Thus, immune cells directly contribute to bone remodelling, and the bone healing process is known to be negatively affected by oestrogen deficiency in elderly women, as oestrogen promotes osteoclastic activity. Many experimental studies have also shown delayed wound healing (impaired bone healing) in ovariectomised rats $[9,22,31,37]$.

The treatment of bone defects is particularly controversial in the case of osteoporosis. The bone grafting procedure is thought to be the most widely used method to enhance bone regeneration and repair bone defects, but it has certain drawbacks, including risks during collection, haemorrhage, infection, chronic pain, sterilisation, storage, especially foreign body reactions, and disease transmission [10, 26]. Therefore, a more effective treatment is needed to improve bony defects in osteoporosis. Mesenchymal stem cells (MSCs) have the ability to differentiate into osteoblasts and are available from a wide variety of sources. Tissue regeneration using autologous stem cells to form a suitable scaffold is an alternative to using autografts and allografts [17]. The bone-regeneration potential of MSCs has been evaluated in bone defects in animals with or without scaffolds $[8,34]$. Currently, different methods for efficient tissue regeneration are being developed with various combinations of stem cells and scaffolds [4, 33].

In light of this information, we hypothesised that treatment with bone marrow (BM)-MSCs combined with bone grafts would facilitate bone repair in osteoporotic bone damage conditions. There is little information available about the healing capacity of BM-MSCs used in combination with demineralised freeze-dried bone allografts (DFDBAs) for the treatment of calvarial bone defects. For this reason, we aimed to evaluate the effects of BM-MSCs and allografts on bone healing in ovariectomised rats via the expression of immunohistochemical markers.

\section{MATERIALS AND METHODS}

\section{Animals}

A total of 48 female Wistar rats (250-300 g) provided by Scientific Application and Research Centre of Dicle University (Protocol No: 12-DH-53) were used. All of the procedures involved in the experimental protocols were approved by the Animal Ethics Committee of Dicle University (Protocol No. 2011/15). The study was performed in accordance with the Helsinki Declaration and with the permission of the Governmental Animal Protection Committee. Because 6 animals died, the study was conducted with 42 rats. Whole animals were provided with commercial rat chow and water ad libitum and were maintained on a $12 \mathrm{~h}$ light/12 $\mathrm{h}$ dark cycle at a temperature of $22 \pm 1^{\circ} \mathrm{C}$.

The animals were anaesthetised by the intraperitoneal administration of xylazine and ketamine and then subjected to ovariectomy. Ovariectomy was preceded by a $3 \mathrm{~cm}$ long midline dorsal skin incision, approximately halfway between the middle of the back and the base of the tail, according to the method described by Pires-Oliveira et al. [23]. The animals were monitored for infection.

Thirty days following ovariectomy, all animals were anaesthetised for the introduction of calvarial bone defects. After the head hair was shaved, a longitudinal midsagittal skin incision was made to expose the parietal bones, and flaps were retracted in a subperiosteal plane, exposing the parietal bones. A 4-mm-diameter full-thickness round-sized cranial defect was made unilaterally in the parietal bone using trephine dental drills with saline water irrigation. Care was taken to avoid injury to the dura in all animals [3]. 
The animals were randomised. The two groups were separated into three subgroups $(n=7)$. Group 1 (control group) was divided into $\mathrm{C} 1$ - animals with cranial defect only, C2 - animals with cranial defect with MSCs, and C3 - animals with DFDBAs with MSCs. Group 2 (ovariectomy group) was divided into $\mathrm{O} 1$ - animals with cranial defect only, $\mathrm{O} 2$ - animals with cranial defect with MSCs, and $\mathrm{O} 3$ - animals with DFDBAs with MSCs. Eight weeks after the calvarial surgery, all of the rats were euthanised with an intraperitoneal overdose of ketamine hydrochloride for histological evaluation.

\section{Isolation and culture of rBM-MSCs}

The isolation and culturing of rat bone marrow mesenchymal stem cells (rBM-MSCs) were performed in vitro according to a published protocol [14]. MSCs were isolated from the bone marrow of rats. Under sterile conditions, femurs and tibias were excised from each rat, bone marrow cells were isolated by flushing the bone marrow cavity with complete medium (L-DMEM supplemented with $10 \%$ foetal bovine serum [FBS, Gibco/Life Technologies] and 1\% penicillin/ /streptomycin) delivered through a 21 gauge needle. After washing, the isolated bone marrow cells were cultured in complete medium at $37^{\circ} \mathrm{C}$ in a humidified atmosphere of $5 \% \mathrm{CO}_{2}$ for 3 days. The unattached cells were removed, and the adhered cells were continually cultured until reaching $70-80 \%$ confluence. The cells were trypsinised and passaged at a ratio of $1: 2$ or $1: 3$. The third-passage rBM-MSCs were pooled and used for characterisation and treatment.

\section{Characterisation of rBM-MSCs}

Undifferentiated rBM-MSCs were subjected to flow cytometry analysis (FACS Calibur [BD Biosciences, San Jose, CA]). The cell suspension was spun at 1000 RPM for $5 \mathrm{~min}$ and the supernatant was decanted. The pellet was resuspended in $1 \mathrm{X}$ phosphate buffered saline (PBS). The cells were counted with a haemocytometer. The desired total number of cells was added to a flow tube (0.5-1 $\times 10^{6}$ per sample). The cells were washed by adding $\sim 1 \mathrm{~mL} 1 \mathrm{X}$ PBS to the flow tube. The cell suspension was spun at 1000 RPM for $5 \mathrm{~min}$ and the supernatant was decanted. The tube was gently tapped to loosen the cell pellet. An appropriate amount of staining buffer ( $50 \mu \mathrm{L}$ per $1 \times 10^{6}$ cells) was added and $1 \times 10^{6}$ cells $(50 \mu \mathrm{L})$ were added to the desired number of flow tubes. Finally, immunophenotyping analysis was performed for the antigens CD29, CD45, CD54, CD90, CD106, MHC class-I and MHC class-II (BD Biosciences).

\section{In vitro differentiation of rBM-MSCs}

Adipogenic and osteogenic differentiation was performed in vitro according to a published protocol [2]. Adipogenic differentiation was performed by incubating rBM-MSCs with L-DMEM supplemented with $0.5 \mathrm{mM}$ isobutyl-methylxanthine, $10^{-6} \mathrm{M}$ dexamethasone, $10 \mu \mathrm{g} / \mathrm{mL}$ insulin and $200 \mu \mathrm{M}$ indomethacin for 2 weeks. The medium was refreshed every 3-4 days. The formation of intracellular lipid droplets, which indicates adipogenic differentiation, was confirmed by staining with $0.5 \%$ oil red O (Sigma-Aldrich, St. Louis, $\mathrm{MO})$. For osteogenic differentiation, the cells were cultured with L-DMEM supplemented with $100 \mathrm{nM}$ dexamethasone, $0.05 \mu \mathrm{M}$ ascorbate-2-phosphate, and $10 \mathrm{mM} \beta$-glycerophosphate for 4 weeks. After 4 weeks, osteogenic differentiation was assessed via staining with $2 \%$ alizarin red $\mathrm{S}(\mathrm{pH} 4.1-4.3$; Fluka, Buchs, Switzerland).

\section{Green fluorescent protein (GFP) labelling of rBM-MSCs}

Mesenchymal stem cells were transfected with pGFP-N (Clontech, Palo Alto, CA) by electroporation (Neon Transfection System, Invitrogen, Carlsbad, CA) following the instructions provided by the manufacturer. After transfection, the cells were cultured with L-DMEM (supplemented with $10 \%$ FBS), and the transformed cells were selected with G418 (Gibco/Life Sciences; $200 \mu \mathrm{g} / \mathrm{mL}$ ) under standard culture conditions for $48 \mathrm{~h}$. GFP-positive cells were maintained in the same medium supplemented with G418 $(200 \mu \mathrm{g} / \mathrm{mL})$ for three passages. The number of GFP + cells was monitored by flow cytometry; $>90 \%$ of the cells used in the treatment were GFP positive.

\section{Immunohistochemical analysis of GFP + rBM-MSCs in the tissue}

Consecutive sections, each $4 \mu \mathrm{m}$ thick, were taken from each paraffin-embedded tissue. To detect GFP + rBM-MSCs, an immunofluorescence staining protocol was performed. Slides were deparaffinised with xylene for 5 min twice and rehydrated in a series of graded alcohol solutions ( $70 \%$ to $100 \%$ ). Endogenous peroxidases were inhibited by incubation with 3\% $\mathrm{H}_{2} \mathrm{O}_{2}$ in PBS buffer. For antigen retrieval, the samples were heated to $98-99^{\circ} \mathrm{C}$ in antigen retrieval buffer (10 mM sodium citrate, $0.05 \%$ Tween $20, \mathrm{pH} 6.0$ ) and 
Table 1. Details of antibodies used

\begin{tabular}{lccccc}
\hline Antibody & Clone, isotype & Host & Cellular localisation & Dilution & Supplier (Catalogue no.) \\
\hline Osteopontin/OPN (human) & AKm2A1, monoclonal lgG & Mouse & Cytoplasmic & $1: 200$ & Santa Cruz (sc-21742) \\
Osteonectin/Sparc (human) & H-90, polyclonal lgG & Rabbit & Cytoplasmic & $1: 200$ & Santa Cruz (sc-25574) \\
Osteocalcin (human) & FL-100, polyclonal lgG & Rabbit & Cytoplasmic & $1: 200$ & Santa Cruz (sc-30044) \\
\hline
\end{tabular}

incubated for $30 \mathrm{~min}$ in a pressurised vessel. Nonspecific staining was blocked with a mixture of $1.5 \%$ serum in PBS for 30 min at room temperature, and the sections were incubated with a mouse monoclonal anti-GFP antibody (SC-9996) at a 1:50 dilution for $1 \mathrm{~h}$ at room temperature. After incubation with appropriate fluorophore-conjugated secondary antibodies, the sections were covered with mounting medium containing DAPI (Santa Cruz, Heidelberg, Germany). The cells were investigated under a fluorescence microscope (Leica DMI 4000B, Wetzlar, Germany).

\section{Light microscopy}

Calvarial tissues were removed and fixed in $10 \%$ neutral buffered formaldehyde solution for $48 \mathrm{~h}$ and then decalcified in $10 \%$ ethylene diaminetetraacetic acid (EDTA) prepared in $0.1 \mathrm{M}$ Tris- $\mathrm{HCl}$ buffer $(\mathrm{pH}$ 7.4) for 14 days. The tissues were subsequently dehydrated, cleared and embedded in paraffin blocks. Five-micrometre-thick sections were cut from these blocks and stained using Gomori's method to determine ossification.

\section{Immunohistochemical staining}

Immunohistochemical investigations were performed on tissue preparations embedded in paraffin and by using a Zymed Histostain Plus Bulk kit (code: 85-9043, Histostain Plus Bulk Kit, Zymed, South San Francisco, CA, USA) and streptavidin-peroxidase [2]. Briefly, sections were deparaffinised, rehydrated and incubated for 15 min in $3 \% \mathrm{H}_{2} \mathrm{O}_{2}$ in methanol. After the sections were washed in PBS, antigen retrieval was performed by boiling in $0.01 \mathrm{M}$ citric buffer $\mathrm{pH} 6.0$ for $30 \mathrm{~min}$ at $95^{\circ} \mathrm{C}$ using a water bath and by cooling for 20 min prior to immunostaining. Sections were then washed in PBS and incubated in protein blocking solution (Ultra $\mathrm{V}$ Block) for $10 \mathrm{~min}$ at room temperature to prevent nonspecific binding. Subsequently, the preparations were incubated with primary antibodies for $20 \mathrm{~h}$ at $+4^{\circ} \mathrm{C}$ (Table 1 ).

After being washed in PBS, the sections were incubated with biotinylated secondary antibodies for
$20 \mathrm{~min}$ at room temperature and washed in PBS. Subsequently, the preparations were incubated in streptavidin peroxidase conjugate for $20 \mathrm{~min}$ at room temperature and were then washed with PBS. To visualise the reaction, the sections were treated with 3'3-diaminobenzidine (DAB) for 5-15 min. After the reaction developed, the sections were counterstained with Gill's haematoxylin, dehydrated through an alcohol series, cleared in xylene, and finally mounted in entellan. Negative controls were used for the confirmation of the staining. As a negative control, the primary antibodies used for staining were replaced with PBS. Osteopontin (OPN), osteocalcin (OC) and osteonectin (ON) expression in bone tissue was examined microscopically at $\times 200$ magnification. Immunohistochemical staining results were evaluated semi quantitatively. The intensity of positive staining was defined as + weak,++ medium,+++ strong, $+/++$ weak to moderate, and $++/+++$ moderate to strong. The slides were examined and photographed using a Nikon Eclipse E400 (Nikon, Tokyo, Japan) microscope equipped with a digital camera (Nikon Coolpix-4500).

\section{Statistical analysis}

Kruskal-Wallis and Mann-Whitney $U$ tests were used as nonparametric statistical analyses. Values of $p<0.05$ were considered statistically significant.

\section{RESULTS}

Histological findings in calvarial bone tissue

In C1 group (animals with cranial defect only, control group), structures (bone spicules) characterised by intramembranous ossification were observed along the border of the cavity. Furthermore, fibrous tissue was abundant in the cavity and vascularisation (angiogenesis) had occurred in some areas. In 01 group (animals with cranial defect only, ovariectomy group), intramembranous ossification was observed neither in the cavity nor along the border of the cavity, and fibrous tissue was not diffusely distributed, but cellular structures were markedly abundant. No significant revascularisation was observed between 
control (animals with cranial defect only) and ovariectomy group rats (animals with cranial defect only).

Group 01 (animals with cranial defect only, ovariectomy group), intramembranous ossification and osteogenesis had occurred locally, and fibrous tissue was diffuse and regularly distributed. Furthermore, vascularisation was significantly increased. In group $\mathrm{O} 2$ (animals with cranial defect with MSCs, ovariectomy group), intramembranous ossification was observed at a limited level and was localised to the border of the cavity. Furthermore, fibrous tissue was irregularly distributed in only some parts of the cavity. The vascularisation in groups C2 (animals with cranial defect with MSCs, control group) and $\mathrm{O} 2$ (animals with cranial defect with MSCs, ovariectomy group) did not differ.

When compared to those in the other control groups, the fibrous structures of the matrix in group C3 (animals with DFDBAs with MSCs, control group) displayed a compact structure as a result of a bone graft having been placed in the cavity, and these structures were also tightly adhered to the border of the cavity. Intramembranous ossification was observed between the portions of the bone graft. In group $\mathrm{O} 3$ (animals with DFDBAs with MSCs, ovariectomy group), ossification was not observed. The fibrous tissue showed a weak distribution between the bone grafts and along the border of the cavity, in a strip-like formation along the latter. Furthermore, in contrast to those in the other groups, the stem cells had differentiated into adipose cells along the border of the cavity and were present in the form of infiltrating cells (Fig. 1).

\section{Immunohistochemistry for osteopontin}

In C1 group (animals with cranial defect only, control group), the osteoprogenitor cells along the border of the cavity were not immunoreactive for osteopontin. However, while the osteoblasts and extracellular matrix (ECM) in the areas of intramembranous ossification along the border of the cavity showed weak OPN immunoreactivity, the newly formed blood vessels in the cavity were positively stained for OPN (Fig. 2-C1). In 01 group (animals with cranial defect only, ovariectomy group), the normal bone tissue showed positive immunoreactivity for OPN, but OPN immunoreactivity was limited to the blood vessels in the newly formed tissue in the cavity (Fig. 2-01).

In group C2 (animals with cranial defect with MSCs, control group), similar to group C1, the osteoprogenitor cells along the border of the cavity did not show OPN immunoreactivity. Although the osteoblasts in the cavity presented weak OPN staining, OPN expression in the blood vessels was increased (Fig. 2-C2). In group $\mathrm{O} 2$ (animals with cranial defect with MSCs, ovariectomy group), staining for OPN was observed neither in the osteoprogenitor cells along the border of the cavity nor in the osteoblasts in the cavity, but the ECM and blood vessels were positive for OPN (Fig. 2-O2).

In group C3 (animals with DFDBAs with MSCs, control group), the osteoprogenitor cells along the border of the cavity and the newly formed osteoblasts and ECM in the periphery of the bone graft demonstrated moderate OPN immunoreactivity, whereas the blood vessels showed strong OPN immunoreactivity (Fig. 2-C3). In group $\mathrm{O} 3$ (animals with DFDBAs with MSCs, ovariectomy group), the newly formed osteoblasts in the periphery of the bone graft presented moderate immunoreactivity for OPN, whereas the staining of the blood vessels was found to be similar to that observed in group C3 (Fig. 2-O3).

\section{Immunohistochemistry for osteocalcin}

In C1 group (animals with cranial defect only, control group), the osteoprogenitor cells along the border of the cavity did not show immunoreactivity for OC, but the osteoblasts presented weak OC immunoreactivity. The ECM in the border of the cavity showed moderate staining, and the blood vessels stained positively for OC (Fig. 3-C1). In 01 group (animals with cranial defect only, ovariectomy group), the osteoprogenitor cells also showed OC immunoreactivity, and when compared to that of group C1, the OC immunoreactivity of the osteoblasts and ECM was weaker (Fig. 3-01).

Compared with that in the control group, the $\mathrm{OC}$ immunoreactivity of the osteoprogenitor cells along the border of the cavity in group C2 (animals with cranial defect with MSCs, control group) was found to be negative, but the staining of ECM and the blood vessels localised to the border of the cavity and the cavity was more intense. Furthermore, the osteoblasts in the cavity displayed weak immunoreactivity for $\mathrm{OC}$ (Fig. 3-C2). In group $\mathrm{O} 2$ (animals with cranial defect with MSCs, ovariectomy group), the osteoprogenitor cells did not show any OC immunoreactivity, and the osteoblasts displayed weak OC immunoreactivity. The ECM of the border of the cavity stained strongly for $\mathrm{OC}$ and the staining in the cavity was observed to be 


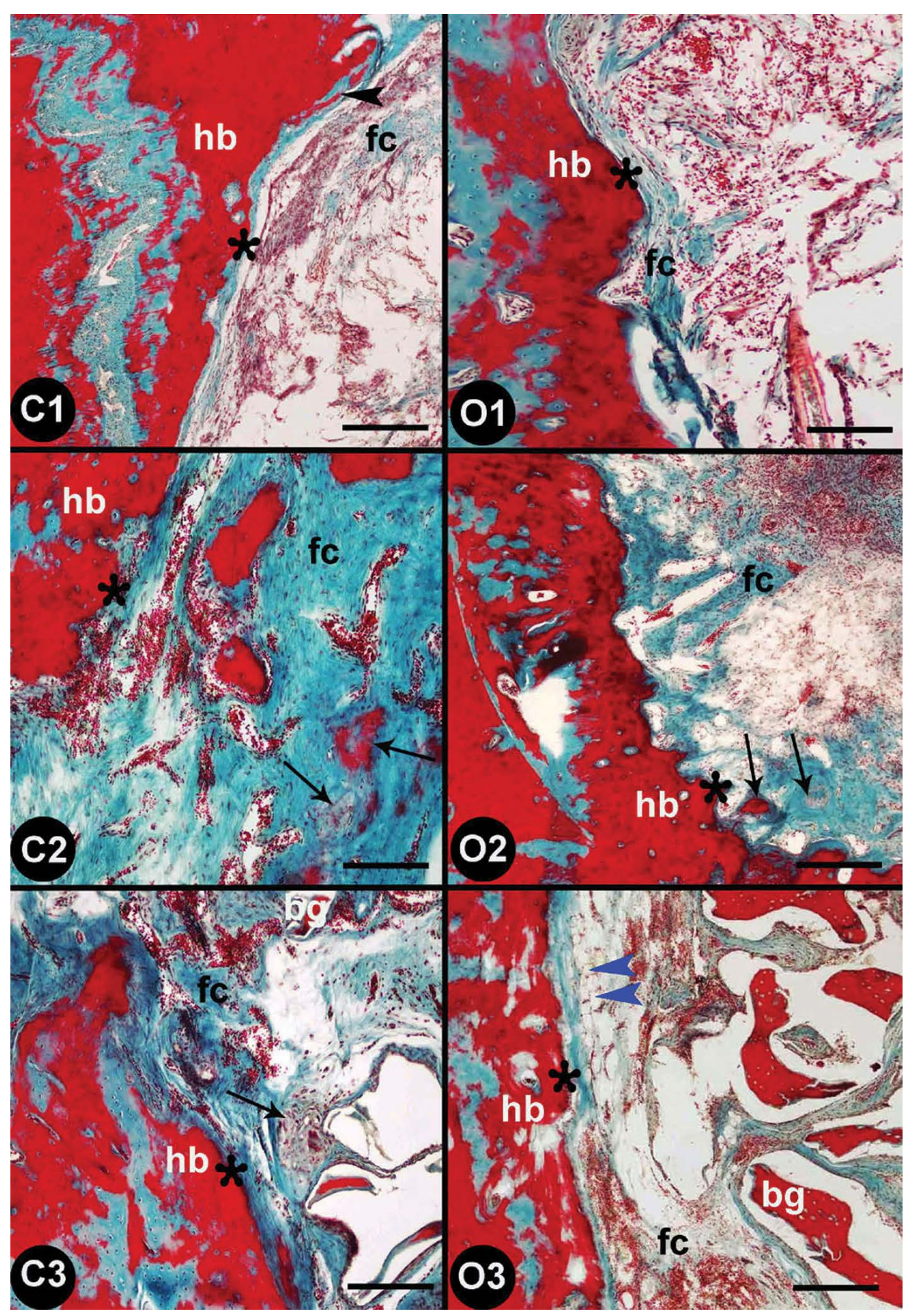

Figure 1. Histological analysis of the defect areas in the rat calvaria bone in the control $(\mathbf{C 1}, \mathbf{C 2}, \mathbf{C 3})$ and ovariectomy $(\mathbf{0 1}, \mathbf{0 2}, \mathbf{0 3})$ groups. The defect was animals with cranial defect only, as shown in panels $\mathrm{C} 1$ and 01 , and the defect areas were treated with stem cells $(\mathrm{C2}, 02)$ and stem cells + bone grafts $(\mathrm{C} 3,03)$; hb - host bone, fc - fibrous connective tissue, bg — bone graft, asterisk — interface between host bone and defect; arrow - ossification areas; black arrowhead — bone spicule; blue arrowhead — adipocytes. Gomori's staining method. Scale bars: $100 \mu \mathrm{m}(\mathrm{C} 1, \mathrm{C} 2$ and 01,02$)$ and $250 \mu \mathrm{m}(\mathrm{C} 3,03)$.

weak. The OC immunoreactivity of the blood vessels was also determined to be strong (Fig. 3-O2).

In group C3 (animals with DFDBAs with MSCs, control group), the newly formed osteoblasts in the periphery of the bone graft displayed OC immunoreactivity ranging from moderate to strong, whereas the osteocytes in the ossified areas displayed weak OC immunoreactivity. The 


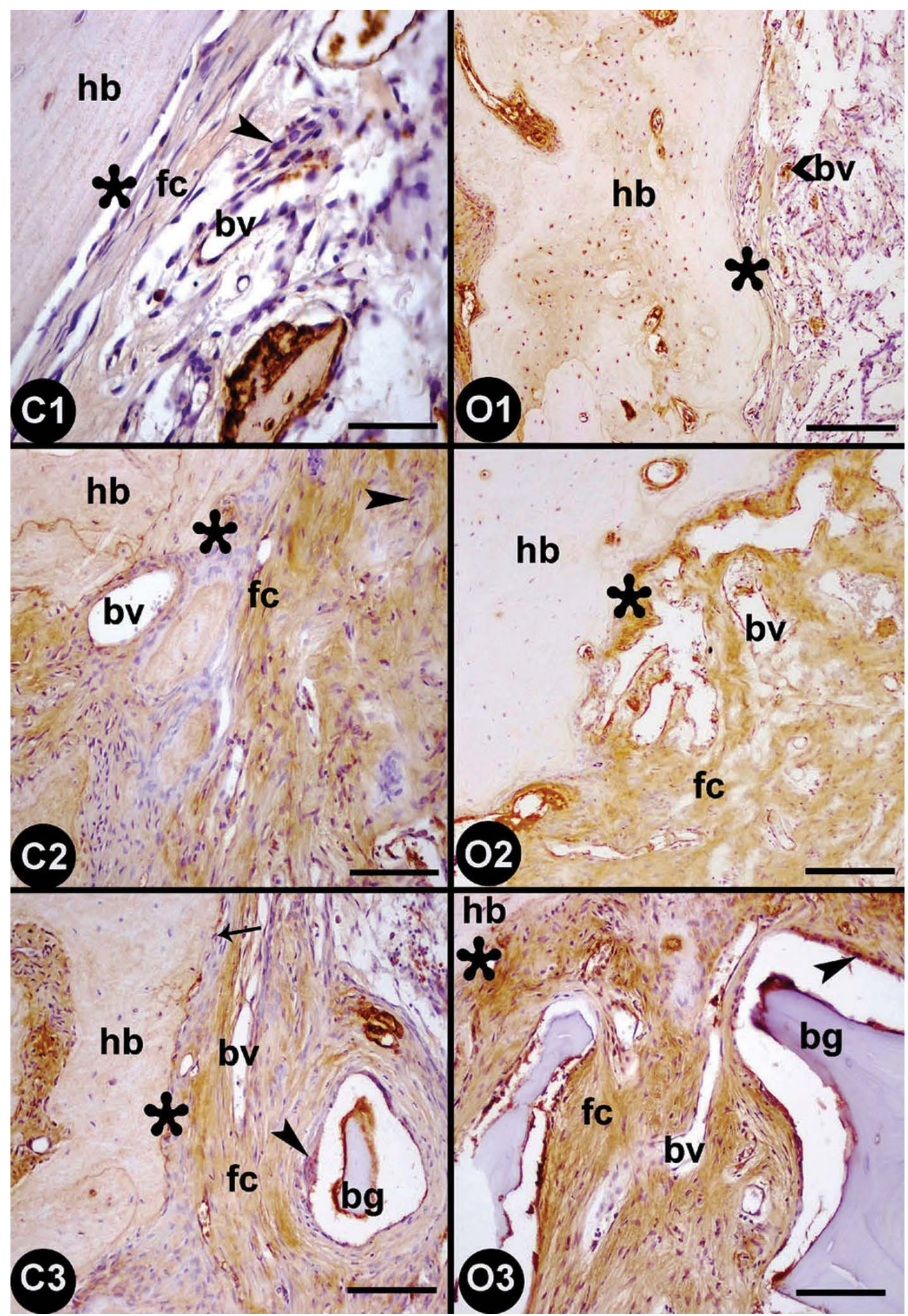

Figure 2. Osteopontin expression in the rat calvaria bone defect areas in the control $(\mathbf{C 1}, \mathbf{C 2}, \mathbf{C 3})$ and ovariectomy $(\mathbf{0 1}, \mathbf{0 2}, \mathbf{0 3})$ groups. The formation of the groups is shown in Figure 1; hb — host bone; fc — fibrous connective tissue; bv — blood vessel; bg — bone graft; asterisk — interface between host bone and defect; arrow — osteoprogenitor cell; arrowhead — osteoblast. Scale bars: $25 \mu \mathrm{m}$ (C1), $50 \mu \mathrm{m}$ (C2, C3, $03)$, and $100 \mu \mathrm{m}(01,02)$.

OC immunoreactivity of the ECM was more homogenous than that in the other groups. Blood vessels also stained positively for OC (Fig. 3-C3). In group 03 (animals with DFDBAs with MSCs, ova- riectomy group), the osteoprogenitor cells displayed negative staining and the osteoblasts and ECM were stained moderately. However, the blood vessels displayed strong immunoreactivity for OC (Fig. 3-O3). 


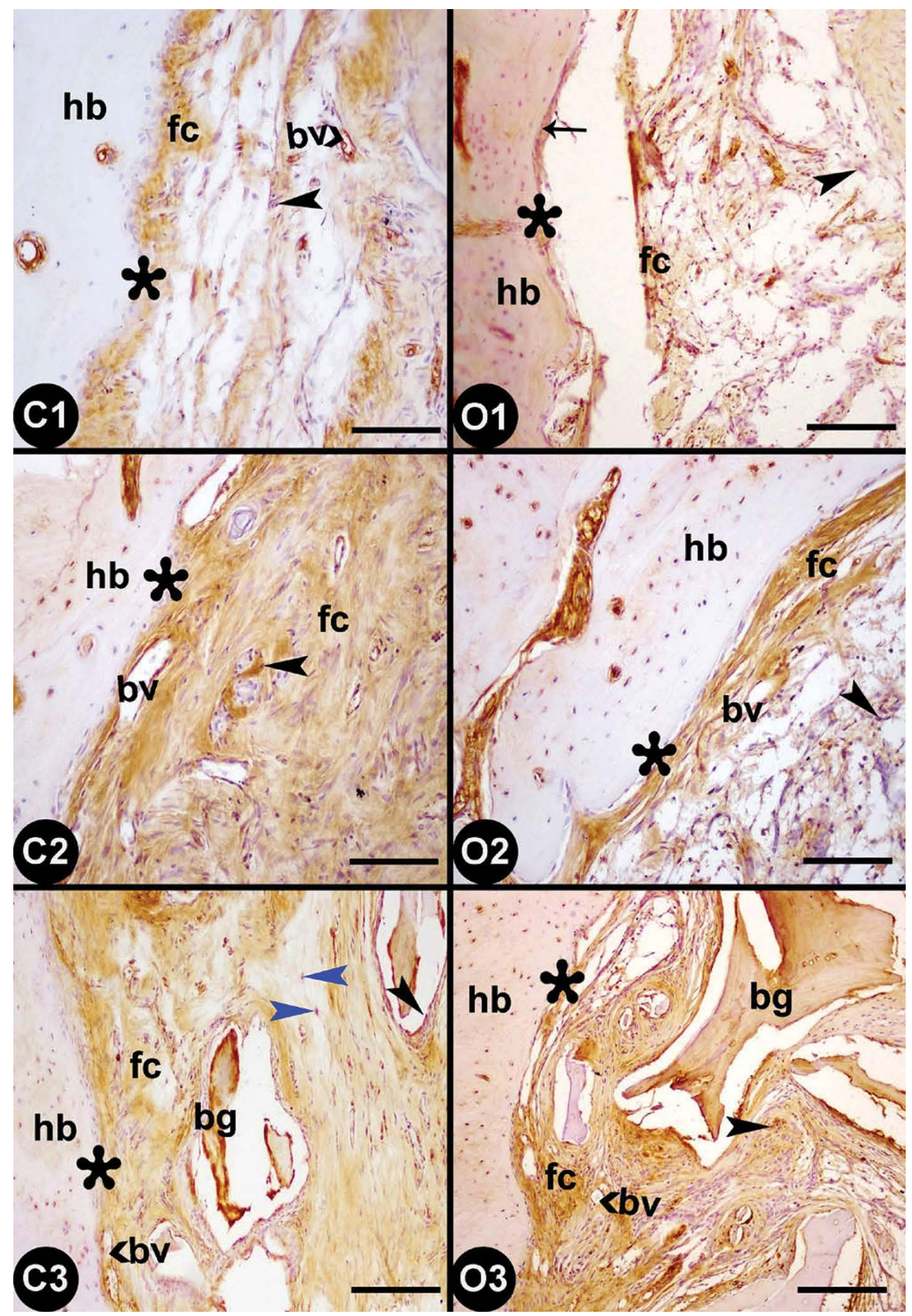

Figure 3. Osteocalcin expression in the rat calvaria bone defect areas in the control $(\mathbf{C 1}, \mathbf{C 2}, \mathbf{C 3})$ and ovariectomy $(\mathbf{0 1}, \mathbf{0 2}, \mathbf{0 3})$ groups. The formation of the groups is shown in Figure 1; hb — host bone; fc — fibrous connective tissue; bv — blood vessel; bg — bone graft; asterisk — interface between host bone and defect; arrow — osteoprogenitor cell; black arrowhead — osteoblast; blue arrowhead — osteocyte. Scale bars: $50 \mu \mathrm{m}(\mathrm{C} 1, \mathrm{C} 2,02)$, and $100 \mu \mathrm{m}(\mathrm{C} 3,01,03)$.

\section{Immunohistochemistry for osteonectin}

In $\mathrm{C} 1$ group (animals with cranial defect only, control group), while the osteoprogenitor cells along the border of the cavity and the osteoblasts in the cavity stained negatively for ON, the ECM showed weak immunoreactivity, and the blood vessels showed moderate immunoreactivity for ON (Fig. 4-C1). Group 01 (animals with cranial defect only, ovariectomy group) showed staining results similar to those of group C1, but the ECM stained weakly (Fig. 4-01). 


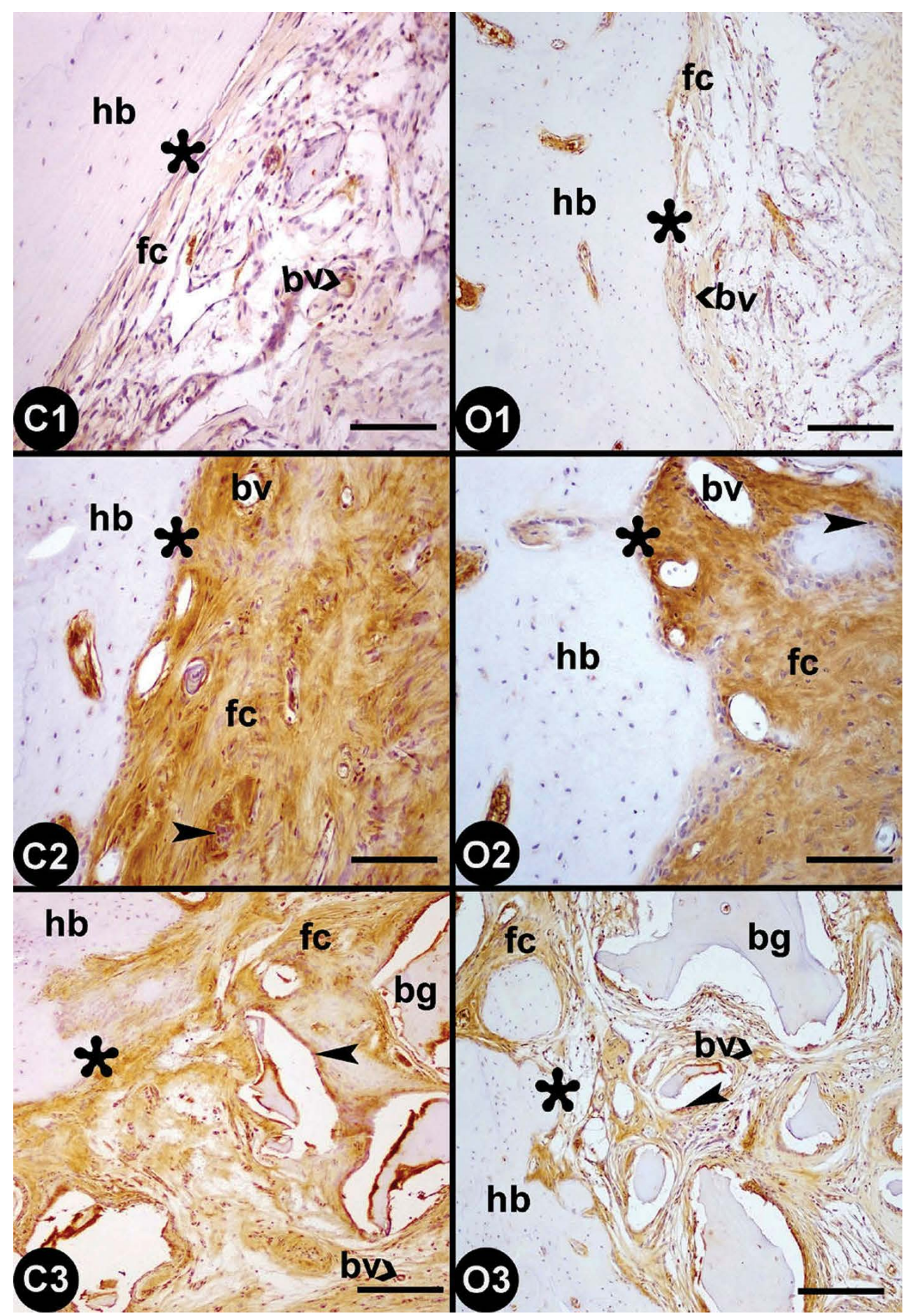

Figure 4. Osteonectin expression in the rat calvaria defect areas in the control $(\mathbf{C 1}, \mathbf{C 2}, \mathbf{C 3})$ and ovariectomy $(\mathbf{0 1}, \mathbf{0 2}, \mathbf{0 3})$ groups. The formation of the groups is shown in Figure 1; hb — host bone; fc — fibrous connective tissue; bv — blood vessel; bg — bone graft; asterisk — interface between host bone and defect; arrowhead — osteoblast. Scale bars: $50 \mu \mathrm{m}(C 1, C 2,02)$ and $100 \mu \mathrm{m}(C 3,01.03)$.

In group C2 (animals with cranial defect with MSCs, control group), the osteoprogenitor cells along the border of the cavity were stained negatively, and the osteoblasts in the cavity showed weak ON expression. In contrast, the ECM and blood vessels were strongly stained (Fig. 4-C2). In group $\mathrm{O} 2$ (animals with cranial defect with MSCs, ovariectomy group), the osteoprogenitor cells along the border of the cavity did not stain, the osteoblasts in the cavity showed weak immunoreactivity for ON. The ECM and 

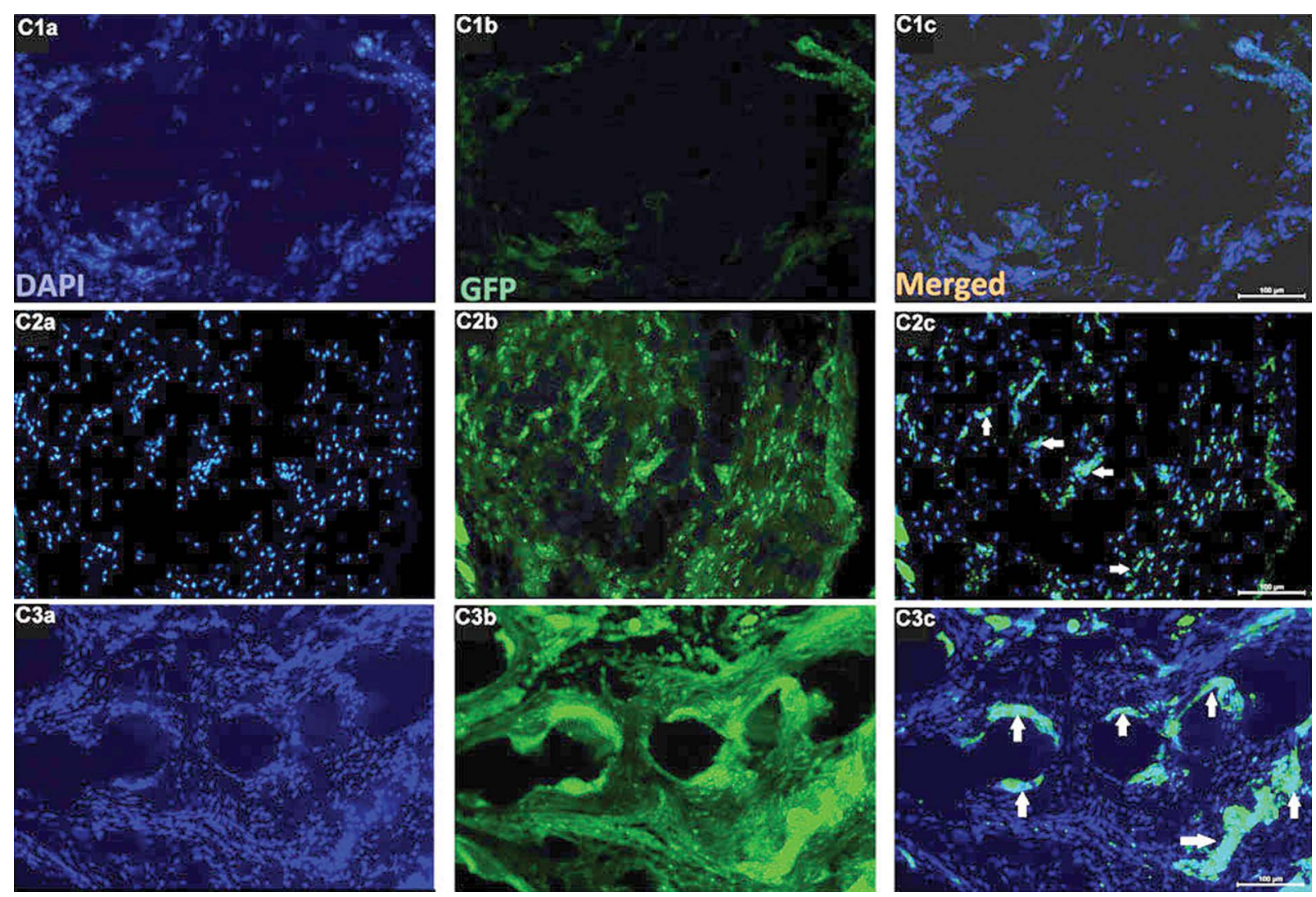

Figure 5. Green fluorescent protein (GFP) fluorescence after immunostaining of the tissue sections from the control group; C1. Animals with cranial defect only; C2. Animals with cranial defect with mesenchymal stem cells (MSCs); C3. Animals with demineralized freeze-dried bone allografts with MSCs. Immune reaction (white arrows) was observed in GFP and cells belonging to C2 and C3 groups. MSCs transplanted in C3 with the graft showed new bone tissue formation. Scale bars: $100 \mu \mathrm{m}$.

blood vessel findings were similar to those of group C2 (Fig. 4-02).

In group C3 (animals with DFDBAs with MSCs, control group), the osteoprogenitor cells along the border of the cavity did not express ON, but the newly formed osteoblasts in the periphery of the bone graft displayed moderate staining. In contrast, the ECM and blood vessels displayed strong ON expression (Fig. 4-C3). In group 03 (animals with DFDBAs with MSCs, ovariectomy group), the osteoprogenitor cells showed no staining and the osteoblasts in the periphery of the bone graft displayed moderate ON expression. It was observed that adipose cells had formed in the cavity, yet these cells displayed no immunoreactivity. However, the ECM and blood vessels showed strong ON immunoreactivity (Fig. 4-O3).

\section{Findings of GFP labelling of RBM-MSCs}

In the control group, no immune reaction was observed for GFP in animals with cranial defect only, without MSCs, whereas GFP and cells (arrows) were observed in C2 (animals with cranial defect with MSCs, control group) and C3 (animals with DFDBAs with MSCs, control group). Animals with DFDBAs in the control group, the MSCs transplanted with the graft show the new tissue formation (Fig. 5). In the ovariectomy group, no immune reaction was observed for GFP in the non-MSCs group (01), whereas GFP and cells (arrows) were observed in $\mathrm{O} 2$ (animals with cranial defect with MSCs, ovariectomy group) and $\mathrm{O} 3$ (animals with DFDBAs with MSCs, ovariectomy group). MSCs transplanted with the graft in $\mathrm{O} 3$ were involved in new tissue formation (Fig. 6).

In addition, the cell expression of each marker is given in Figure 7 for fluorescence intensity.

\section{Statistical findings}

When osteopregenitor cells were evaluated between control (animals with DFDBAs) and ovariectomy groups (animals with DFDBAs), a significant 

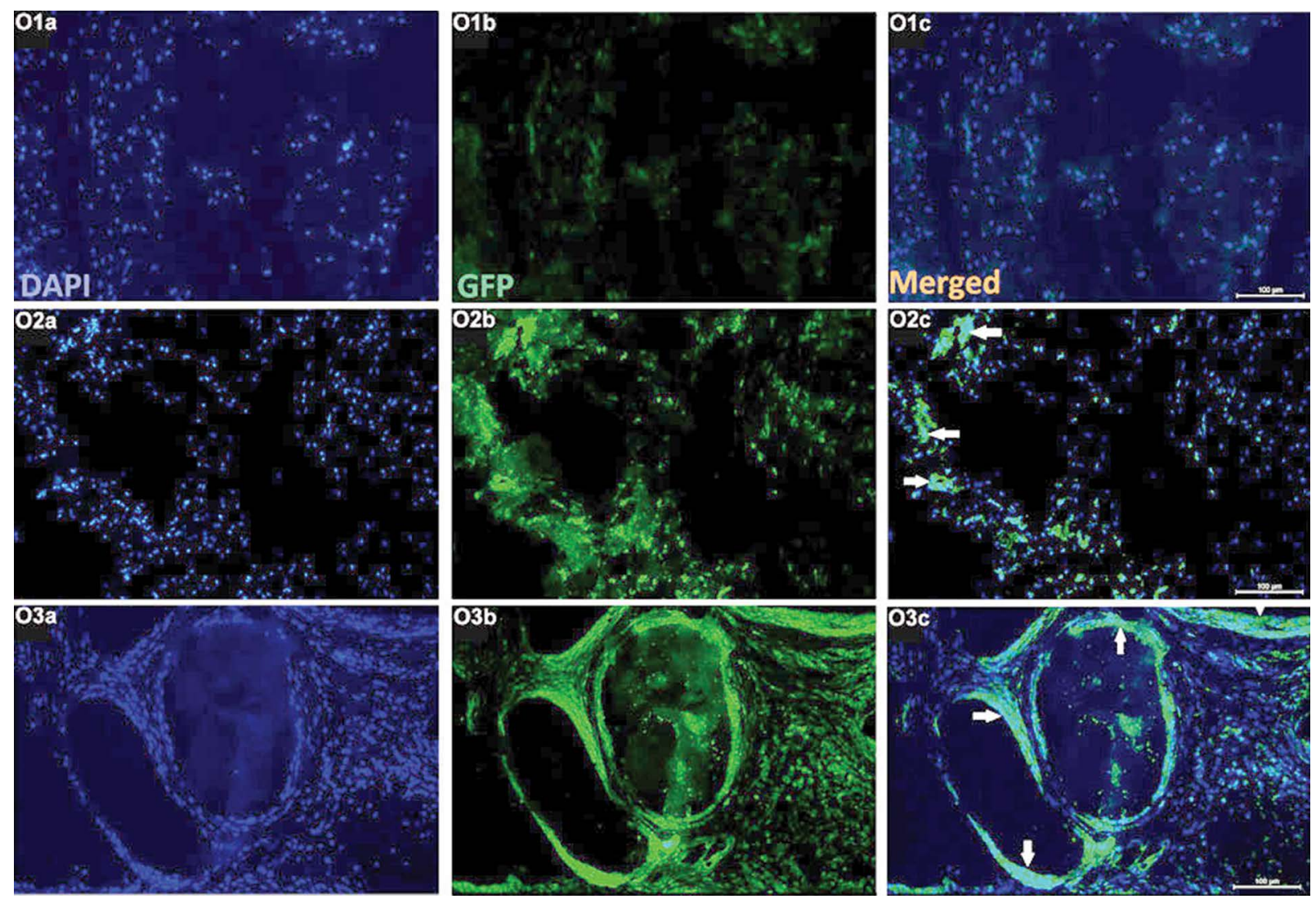

Figure 6. Green fluorescent protein (GFP) fluorescence after immunostaining of the tissue sections from the ovariectomy group; 01. Animals with cranial defect only, 02. Animals with cranial defect with mesenchymal stem cells (MSCs); 03. Animals with demineralized freeze-dried bone allografts with MSCs. Immune reactions (white arrows) were observed in GFP and cells belonging to 02 and 03 groups. Graft transplanted MSCs in 03 appeared to participate in new bone tissue formation. Scale bars: $100 \mu \mathrm{m}$.
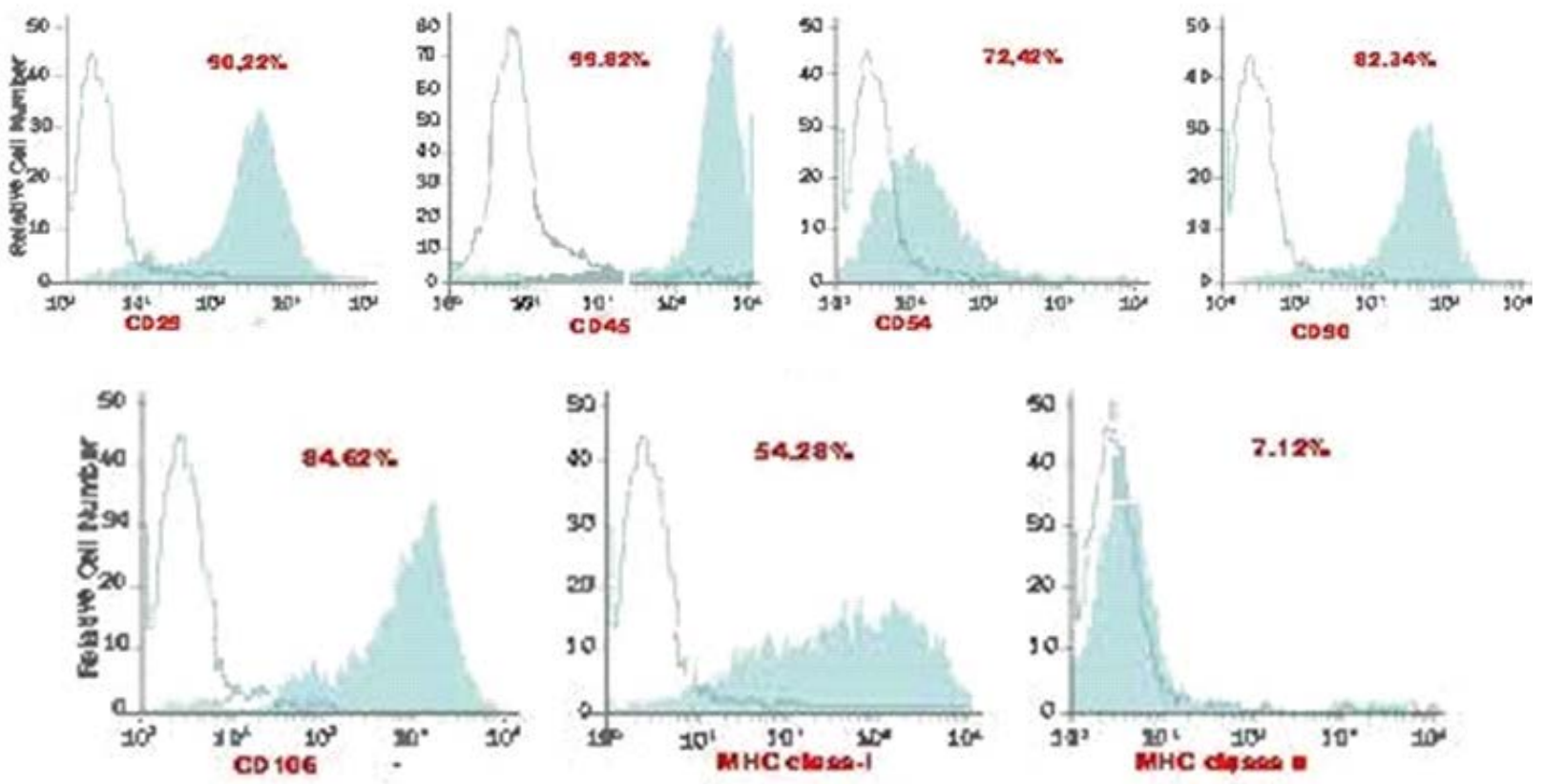

Figure 7. Fluorescence intensity. Numbers in panels represent mean fluorescent intensity of the cells expressing each marker. 
Table 2. Comparisons of the parameters within the groups

\begin{tabular}{|c|c|c|c|c|c|}
\hline Groups & $\mathbf{P}$ & Parameter & $\mathbf{N}$ & Average rank & Different $(\mathrm{p}<0.05$ ) from factor no. \\
\hline (1) C1 & 0.0001 & 1 & 7 & 7.50 & (3) \\
\hline (2) C2 & & & 7 & 7.50 & (3) \\
\hline (3) C3 & & & 7 & 18.00 & $(1)(2)$ \\
\hline (1) $\mathrm{Cl}$ & 0.0109 & 2 & 7 & 7.36 & (3) \\
\hline (2) C2 & & & 7 & 9.71 & (3) \\
\hline (3) C3 & & & 7 & 15.93 & $(1)(2)$ \\
\hline (1) C1 & NS & 7 & 3 & 11.00 & NS \\
\hline (2) C2 & & & 7 & 11.00 & \\
\hline (3) C3 & & & 7 & 11.00 & \\
\hline (1) C1 & 0.0007 & 4 & 7 & 4.86 & (2)(3) \\
\hline (2) C2 & & & 7 & 16.57 & $(1)(3)$ \\
\hline (3) C3 & & & 7 & 11.57 & (1)(2) \\
\hline (1) 01 & NS & 1 & 7 & 11.00 & NS \\
\hline (2) 02 & & & 7 & 11.00 & \\
\hline (3) 03 & & & 7 & 11.00 & \\
\hline (1) 01 & 0.001 & 2 & 7 & 7.50 & (3) \\
\hline (2) 02 & & & 7 & 7.50 & (3) \\
\hline (3) 03 & & & 7 & 18.00 & (1)(2) \\
\hline (1) 01 & NS & 3 & 7 & 11.00 & NS \\
\hline (2) 02 & & & 7 & 11.00 & \\
\hline (3) 03 & & & 7 & 11.00 & \\
\hline (1) 01 & 0.0004 & 4 & 7 & 4.00 & (2)(3) \\
\hline (2) 02 & & & 7 & 15.00 & (1) \\
\hline (3) 03 & & & 7 & 14.00 & (1) \\
\hline
\end{tabular}

NS - non-significant; $p>0.05 ; 1$ - osteopregenitor cells; 2 - osteoblasts; 3 - osteocytes; 4 - extracellular matrix

Table 3. Comparisons of the parameters between the control and test groups

\begin{tabular}{lcccccc}
\hline Parameter & Groups & $\mathbf{P}$ & Groups & $\mathbf{P}$ & Groups & $\mathbf{P}$ \\
\hline 1 & C1 & NS & C2 & NS & C3 & 0.001 \\
& 01 & & 02 & & 03 & \\
2 & C1 & 0.007 & C2 & 0.002 & C3 & NS \\
& 01 & & 02 & & 03 & \\
3 & C1 & NS & C2 & NS & C3 & NS \\
& 01 & & 02 & & 03 & \\
4 & C1 & 0.002 & C2 & NS & C3 & 0.015 \\
& 01 & & 02 & & 03 & \\
\hline
\end{tabular}

NS - non-significant; $p>0.05 ; 1$ - osteopregenitor cells; 2 - osteoblasts; 3 - osteocytes; 4 - extracellular matrix

statistical difference was found (Tables 2, 3). When osteoblast cells were evaluated between control and ovariectomy groups, the difference between $\mathrm{C} 1$ (animals with cranial defect only, control group)-01 (animals with cranial defect only, ovariectomy group) and
C2 (animals with cranial defect with MSCs, control group)-O2 (animals with cranial defect with MSCs, ovariectomy group) groups was found to be statistically significant (Tables 2, 3). And, when the ECM was evaluated, the difference between $\mathrm{C} 1$ (animals 
with cranial defect only, control group)-01 (animals with cranial defect only, ovariectomy group) and C3 (animals with DFDBAs with MSCs, control group)-O3 (animals with DFDBAs with MSCs, ovariectomy group) groups was found to be statistically significant (Tables 2, 3).

\section{DISCUSSION}

We aimed to investigate the healing capacity of BM-MSCs used with DFDBAs for the treatment of calvarial bone defects, assuming that BM-MSC combined with bone grafts would facilitate bone repair under conditions of osteoporotic bone injury. Therefore, the effects of BM-MSCs and allografts on bone healing in ovariectomised rats were concluded by evaluating immunohistochemical results.

Sethi et al. [28] evaluated and interpreted both clinically and radiographically by studying the changes post 1 week, 1 month, 3 months, and 6 months, respectively. It was stated that there was evidence of trabecular formation and calcification. They concluded that platelet-rich plasma-enriched DFDBA was a superior inoculant in terms of other available inoculants in patients [28]. In this experimental study, we observed the successful results of DFDBAs. So called "mesenchymal stem cell chondrocytes technique" was used to reconstruct a $15 \mathrm{~mm}$ massive femoral defect (approximately $50 \%$ of rat femur shaft length) in an experimental study. According to their results, considering the high repairability and the excellent biomechanical forces of the repaired femora, they concluded that the reconstruction of the large bone defect may be possible [36].

In an experimental study in which $8 \mathrm{~mm}$ defect was applied to one of the groups in which decalcified freeze-dried bone allograft was applied, statistically significant results were obtained when compared with the other groups [20]. In an experimental study, ovariectomy was performed methodologically as in our study. It has been stated that limited proteins are known to be involved in the subsequent stages of bone formation and maturation in the proteomes of animals [6].

As a result of these cellular changes, osteogenesis becomes impaired and the bone formation period is insufficient to repair the increased bone destruction in oestrogen deficiency-related osteoporosis [18, 21]. He et al. [9] also found that the femurs of mice with ovariectomy-induced osteoporosis showed impaired angiogenesis, osteogenesis, and remodelling in their study [9]. Calciolari et al. [6] observed immature bone formation both in ovariectomy and control calvarial CSDs over a 30-day period [6]. Stockmann et al. [29] also observed a similar result during the early stage of bone healing on the $30^{\text {th }}$ day. There were no differences between the test and control groups, but at 60 days, new bone formation was achieved in the MSC group; however, significant pig calvarial bone regeneration was measured at day 90 [29]. In our study, we observed intramembranous ossification in animals with cranial defect only of the healthy control rats, after 60 days. Consistent with other ovariectomy-induced osteoporosis rat model studies, we did not observe intramembranous ossification either in the cavity or along the border of the cavity of animals with cranial defect only with ovariectomy group after 8 weeks. However, in cortical bones, such as the calvarial bone, the healing process is slower than that in cancellous bone, with a poorer blood supply and less bone marrow; thus, much more than 60 days might be necessary for mature bone formation.

Recently, it has been stated that the advantages and disadvantages of autogenous, allogeneic, xenogenic and alloplastic materials have gained meaning in periodontal treatment [30]. Kurkalli et al. [16] concluded that the placement of the osteogenic composite in a large deficient area of the parietal bone of the skull of rats resulted in a large demineralised bone matrix particle structure, fully reconstituted haematopoietic microenvironment within 30 days, and a well-integrated normal smooth bone [16]. Intini et al. [11] found that demineralised freeze-dried bone was not effective enough to induce bone formation in rat calvaria 8 weeks after surgery [11]. Caplanis et al. [7] did not find any histological effects on bone formation in canine defects treated with DFDBAs after 4 weeks [7]. Bertolai et al. [5] have successfully used freeze-dried bone as a graft material in the treatment of maxillary atrophy, as in our study [5].

In the meta-analysis, the authors concluded that MSCs improved bone regeneration, and it is preferable to use MSCs with an appropriate scaffold. Koob et al. [15] mentioned that MSCs enhance bone formation in calvarial defects. Moreover, in large animal studies, autologous MSCs transplanted alone or in combination with different bone substitutes were found to significantly increase bone formation in critical-sized defects [10]. Kandal et al. [13] suggested that the combined use of demineralised bone matrix with MSCs increases the osteoinductive responses 
in the frontal bone of rats. They suggested that this combination can provide enhanced craniofacial bone reconstruction results at the end of 12 weeks [13]. In another experimental study, the utilisation of MSCs with platelet-rich plasma and synthetic bone substitutes was found to enhance new bone formation [1]. Semyari et al. [27] observed the overall recovery of a bony defect treated with MSCs on different scaffolds with membranes after 8 weeks of calvarial surgery in rabbits.

Osteopontin has been implicated as being an important factor in bone remodelling. Research suggests it plays a role in attaching osteoclasts to the mineral matrix of bones and in the regulation of normal mineralisation within the extracellular matrices of bones and teeth. Osteocalcin and osteonectin are not observed during initial crystal formation but are seen in the later stages of bone formation [32]. Therefore, we chose these three bone markers to assess the bone formation activity. We also observed the effect of oestrogen deficiency on bone healing via the expression of these bone markers, as there are few studies on this issue. In our study, the osteoblasts and ECM staining for these proteins was weak in animals with cranial defect only in the control and ovariectomy groups without MSCs. Histological and immunohistochemical evaluation revealed that the findings obtained for the ECM, ossification and blood vessels were similar between groups $\mathrm{O} 2$ (animals with cranial defect with MSCs, ovariectomy group) and C2 (animals with cranial defect with MSCs, control group). Thus, oestrogen deficiency may not influence the expression of bone markers, which is consistent with the findings of Tera de Mellod et al. [32]. According to the results of this study, more new bone formation was observed in defects treated with MSCs alone than was observed in animals with cranial defect only. However, the combination of DFDBA/MSCs in animals with DFDBAs of ovariectomy group was not as effective on compact intramembranous ossification at the end of the 8 weeks as expected.

Akita et al. [3] found that there were no significant differences in $4 \mathrm{~mm}$ cranial rat defects among groups treated with MSCs only or MSCs with FGF-BMP at 8 weeks after transplantation. Similar to our study, Wang et al. [35] created bony defects in ovariectomised rabbits and treated the defects with MSCs/decalcified bone matrix. Three months later, the authors concluded that the defect treatment was ineffective for the osteoporotic state and that the bone formation was significantly worse than that of the control group [35].

The properties of scaffolds are important for the migration, proliferation and differentiation of living cells during bone regeneration. In this study, the combination of human (DFDBAs) and animal (rBM-MSCs) scaffolds may be biologically incompatible. However, the osteogenic potential of the DFDBA may be diminished during the production process. Additionally, bone healing may have been negatively affected by the absence of a collagen membrane in the scaffold.

\section{Limitations of the study}

There are some limitations to the current study. It would be better to evaluate bone formation with histomorphometric parameters than histochemical staining. In addition, a collagen membrane may be used with the scaffold for 12 weeks to achieve complete bone regeneration.

\section{CONCLUSIONS}

In conclusion, stem cell therapy could be an option to manage impaired bone formation. However, to achieve compact bone formation it is preferential to use proper scaffolds loaded with BM-MSCs for the appropriate healing time. Because there are limited studies in this field, further studies are required to investigate the proliferation and differentiation of MSCs in different scaffolds for the enhancement of impaired bone formation.

\section{Acknowledgements}

This work was supported by the Scientific Application and Research Centre of Dicle University (Protocol No: 12-DH-53).

\section{REFERENCES}

1. Agacayak S, Gulsun B, Ucan MC, et al. Effects of mesenchymal stem cells in critical size bone defect. Eur Rev Med Pharmacol Sci. 2012; 16(5): 679-686, indexed in Pubmed: 22774411.

2. Akbalik ME, Ketani MA. Expression of epidermal growth factor receptors and epidermal growth factor, amphiregulin and neuregulin in bovine uteroplacental tissues during gestation. Placenta. 2013; 34(12): 1232-1242, doi: 10.1016/j. placenta.2013.09.019, indexed in Pubmed: 24138732.

3. Akita S, Fukui M, Nakagawa $\mathrm{H}$, et al. Cranial bone defect healing is accelerated by mesenchymal stem cells induced by coadministration of bone morphogenetic protein-2 and 
basic fibroblast growth factor. Wound Repair Regen. 2004; 12(2): 252-259, doi: 10.1111/j.1067-1927.2004.012118.x, indexed in Pubmed: 15086777.

4. Alfotawei R, Naudi KB, Lappin D, et al. The use of TriCalcium Phosphate (TCP) and stem cells for the regeneration of osteoperiosteal critical-size mandibular bony defects, an in vitro and preclinical study. J Craniomaxillofac Surg. 2014; 42(6): 863-869, doi: 10.1016/j.jcms.2013.12.006, indexed in Pubmed: 24485270.

5. Bertolai R, Catelani $C$, Aversa A, et al. Bone graft and mesenchimal stem cells: clinical observations and histological analysis. Clin Cases Miner Bone Metab. 2015; 12(2): 183-187, doi: 10.11138/ccmbm/2015.12.2.183, indexed in Pubmed: 26604947.

6. Calciolari E, Mardas N, Dereka X, et al. The effect of experimental osteoporosis on bone regeneration: part 2, proteomics results. Clin Oral Implants Res. 2017; 28(9): e135-e145, doi: 10.1111/clr.12950, indexed in Pubmed: 27580862.

7. Caplanis N, Lee MB, Zimmerman GJ, et al. Effect of allogeneic freeze-dried demineralized bone matrix on regeneration of alveolar bone and periodontal attachment in dogs. J Clin Periodontol. 1998; 25(10): 801-806, doi: 10.1111/j.1600-051x.1998.tb02373.x, indexed in Pubmed: 9797052.

8. Gamie Z, Tran GT, Vyzas G, et al. Stem cells combined with bone graft substitutes in skeletal tissue engineering. Expert Opin Biol Ther. 2012; 12(6): 713-729, doi: 10.1517/14712598.2012.679652, indexed in Pubmed: 22500826.

9. He $Y X$, Zhang Ge, Pan $X H$, et al. Impaired bone healing pattern in mice with ovariectomy-induced osteoporosis: A drill-hole defect model. Bone. 2011; 48(6): 1388-1400, doi: 10.1016/j.bone.2011.03.720, indexed in Pubmed: 21421090.

10. Im JY, Min WK, You C, et al. Bone regeneration of mouse critical-sized calvarial defects with human mesenchymal stem cells in scaffold. Lab Anim Res. 2013; 29(4): 196-203, doi: 10.5625/lar.2013.29.4.196, indexed in Pubmed: 24396384.

11. Intini G, Andreana S, Buhite RJ, et al. A comparative analysis of bone formation induced by human demineralized freeze-dried bone and enamel matrix derivative in rat calvaria critical-size bone defects. J Periodontol. 2008; 79(7): 1217-1224, doi: 10.1902/jop.2008.070435, indexed in Pubmed: 18597604.

12. Juluri R, Prashanth E, Gopalakrishnan D, et al. Association of postmenopausal osteoporosis and periodontal disease: a double-blind case-control study. J Int Oral Health. 2015; 7(9): 119-123, indexed in Pubmed: 26435630.

13. Kandal S, Özmen S, Uygur S, et al. Effects of rat bone marrow-derived mesenchymal stem cells and demineralized bone matrix on cranial bone healing. Ann Plast Surg. 2016; 77(2): 249-254, doi: 10.1097/SAP.0000000000000274, indexed in Pubmed: 27070677.

14. Karaoz E, Aksoy A, Ayhan S, et al. Characterization of mesenchymal stem cells from rat bone marrow: ultrastructural properties, differentiation potential and immunophenotypic markers. Histochem Cell Biol. 2009; 132(5): 533-546, doi: 10.1007/s00418-009-0629-6, indexed in Pubmed: 19688349.
15. Koob S, Torio-Padron N, Stark GB, et al. Bone formation and neovascularization mediated by mesenchymal stem cells and endothelial cells in critical-sized calvarial defects. Tissue Eng Part A. 2011; 17(3-4): 311-321, doi: 10.1089/ ten.TEA.2010.0338, indexed in Pubmed: 20799886.

16. Kurkalli BG, Gurevitch O, Sosnik A, et al. Repair of bone defect using bone marrow cells and demineralized bone matrix supplemented with polymeric materials. Curr Stem Cell Res Ther. 2010; 5(1): 49-56, doi: 10.2174/157488810790442831, indexed in Pubmed: 19807659.

17. Lu W, Ji K, Kirkham J, et al. Bone tissue engineering by using a combination of polymer/Bioglass composites with human adipose-derived stem cells. Cell Tissue Res. 2014; 356(1): 97-107, doi: 10.1007/s00441-013-1770-z, indexed in Pubmed: 24408074.

18. McCann RM, Colleary G, Geddis C, et al. Effect of osteoporosis on bone mineral density and fracture repair in a rat femoral fracture model. J Orthop Res. 2008; 26(3): 384-393, doi: 10.1002/jor.20505, indexed in Pubmed: 17960650.

19. Miron RJ, Wei L, Yang S, et al. Effect of enamel matrix derivative on periodontal wound healing and regeneration in an osteoporotic model. J Periodontol. 2014; 85(11): 1603-1611, doi: 10.1902/jop.2014.130745, indexed in Pubmed: 24857323.

20. Mokbel N, Bou Serhal C, Matni G, et al. Healing patterns of critical size bony defects in rat following bone graft. Oral Maxillofac Surg. 2008; 12(2): 73-78, doi: 10.1007/ s10006-008-0107-7, indexed in Pubmed: 18618164.

21. Namkung-Matthai $H$, Appleyard R, Jansen J, et al. Osteoporosis influences the early period of fracture healing in a rat osteoporotic model. Bone. 2001; 28(1): 80-86, doi: 10.1016/s8756-3282(00)00414-2.

22. Palomo L, Williams K, Thacker H. Periodontal healing and osteoporosis in postmenopausal women. Ann Gerontol Geriatric Res. 2016; 3(3): 1043.

23. Pires-Oliveira DAA, Oliveira RF, Amadei SU, et al. Laser $904 \mathrm{~nm}$ action on bone repair in rats with osteoporosis. Osteoporos Int. 2010; 21(12): 2109-2114, doi: 10.1007/ s00198-010-1183-8, indexed in Pubmed: 20204601.

24. Richa RY, Puranik MP, Shrivastava A, et al. Association between osteoporosis and periodontal disease among postmenopausal Indian women. J Investig Clin Dent. 2017; 8(3), doi: 10.1111/jicd.12223, indexed in Pubmed: 27339765.

25. Riggs $B L$. The mechanisms of estrogen regulation of bone resorption. J Clin Invest. 2000; 106(10): 1203-1204, doi: 10.1172/JCl11468, indexed in Pubmed: 11086020.

26. Saad KAE, Abu-Shahba AG, El-Drieny EAE, et al. Evaluation of the role of autogenous bone-marrow-derived mesenchymal stem cell transplantation for the repair of mandibular bone defects in rabbits. J Craniomaxillofac Surg. 2015; 43(7): 1151-1160, doi: 10.1016/j.jcms.2015.04.013, indexed in Pubmed: 26048107.

27. Semyari H, Rajipour M, Sabetkish S, et al. Evaluating the bone regeneration in calvarial defect using osteoblasts differentiated from adipose-derived mesenchymal stem cells on three different scaffolds: an animal study. Cell Tissue Bank. 2016; 17(1): 69-83, doi: 10.1007/s10561015-9518-5, indexed in Pubmed: 26108195.

28. Sethi AK, Kar IB, Mohanty T, et al. Use of plasma-enriched demineralized freeze-dried bone matrix in postsurgical 
jaw defects. Natl J Maxillofac Surg. 2018; 9(2): 174-183, doi: 10.4103/njms.NJMS_33_18, indexed in Pubmed: 30546232.

29. Stockmann P, Park J, von Wilmowsky C, et al. Guided bone regeneration in pig calvarial bone defects using autologous mesenchymal stem/progenitor cells - a comparison of different tissue sources. J Craniomaxillofac Surg. 2012; 40(4): 310-320, doi: 10.1016/j.jcms.2011.05.004, indexed in Pubmed: 21723141.

30. Sukumar S, Drízhal I. Bone grafts in periodontal therapy. Acta Medica (Hradec Kralove). 2008; 51(4): 203-207, indexed in Pubmed: 19453085.

31. Tarantino U, Cerocchi I, Scialdoni A, et al. Bone healing and osteoporosis. Aging Clin Exp Res. 2011; 23(2 Suppl): 62-64.

32. Tera de Mellod T, Nascimento RD, Prado RF, et al. Immunolocalization of markers for bone formation during guided bone regeneration in osteopenic rats. J Appl Oral Sci. 2014; 22(6): 541-553, doi: 10.1590/1678-775720140190, indexed in Pubmed: 25591022.

33. Vahabi S, Amirizadeh N, Shokrgozar MA, et al. A comparison between the efficacy of Bio-Oss, hydroxyapatite trical- cium phosphate and combination of mesenchymal stem cells in inducing bone regeneration. Chang Gung Med J. 2012; 35(1): 28-37, doi: 10.4103/2319-4170.106169, indexed in Pubmed: 22483425.

34. Viña JA, El-Alami M, Gambini J, et al. Application of mesenchymal stem cells in bone regenerative procedures in oral implantology. A literature review. J Clin Exp Dent. 2014; 6(1): e60-e65, doi: 10.4317/jced.51186, indexed in Pubmed: 24596637.

35. Wang ZX, Chen C, Zhou Q, et al. The treatment efficacy of bone tissue engineering strategy for repairing segmental bone defects under osteoporotic conditions. Tissue Eng Part A. 2015; 21(17-18): 2346-2355, doi: 10.1089/ten. TEA.2015.0071, indexed in Pubmed: 26066049.

36. Watanabe Y, Harada N, Sato K, et al. Stem cell therapy: is there a future for reconstruction of large bone defects? Injury. 2016; 47: S47-S51, doi: 10.1016/s00201383(16)30012-2.

37. Weitzmann MN, Pacifici R. Estrogen deficiency and bone loss: an inflammatory tale. J Clin Invest. 2006; 116(5): 1186-1194, doi: 10.1172/JCI28550, indexed in Pubmed: 16670759. 